\title{
Geopolitik als Literatur - ein germanistischer Beitrag zur Genealogie der deutschen Politischen Geographie und Geopolitik
}

\author{
Niels Werber \\ Neuere deutsche Literaturwissenschaft, Universität Siegen, 57068 Siegen, Germany \\ Correspondence: Niels Werber (werber@germanistik.uni-siegen.de)
}

Received: 30 November 2017 - Revised: 10 May 2018 - Accepted: 22 May 2018 - Published: 19 July 2018

\begin{abstract}
Kurzfassung. This contribution of a literary scholar to the history of geopolitics argues for the consideration of literary sources as constituents of the „classical“ geopolitical discourse (Ratzel, Kjellén, Haushofer). It exemplifies this claim by revealing the geopolitical components through which Gustav Freytag's novel Soll und Haben (Debit and Credit, Freytag, 1855, 1858) creates spaces, civilizations, and stories. Approaching these issues from the perspective of literary history and the history of knowledge, this paper turns to Freytag's novel to examine whether the literature of the period of the so-called „,bourgeois“ or ,poetic" realism belongs to the genealogy of German political geography and geopolitics and how to study its impact on their increasingly manifest political program after the beginning of the First World War. Especially the establishment of the topos of the „Volk ohne Raum" (people without space) in the east of the German Reich and the reorientation of geopolitics ",gen Osten“ (toward the east) in the early twentieth century find a highly compatible antecedent in Soll und Haben.
\end{abstract}

\section{Raum und Literatur}

Der Titel dieses Beitrages, Geopolitik als Literatur, kündigt zwei Thesen an: Erstens kann die Politische Geographie, wie sie etwa in den Werken Friedrich Ratzels und Rudolf Kjelléns Kontur und Resonanz gewinnt, als Literatur gelesen werden, also nicht allein als wissenschaftliche oder doch zumindest parawissenschaftliche Werke, sondern wie Romane oder Erzählungen als literarisch verfasste Texte, deren Landschaftsbeschreibungen, Motive, Handlungselemente, semantische Strukturen literarturwissenschaftlich analysiert werden können. Diese Herangehensweise geht von der Hypothese aus, dass Plausibilität oder Evidenz in diesen Texten einiger der wichtigsten Autoren der deutschen Geopolitik nicht nur mit wissenschaftlichen Methoden hergestellt wird, sondern auch mit literarischen Verfahren und Figuren, die über die notorische rhetorische Verfasstheit von Texten aller Art weit hinausgehen. Der Beitrag kann hier auf ein breites Fundament von Forschungsbeiträgen zurückgreifen, die seit dem sog. „topographical turn“ (um 1990) der Literatur- und Kulturwissenschaften die semantische Konstitution von literarischen Räumen, die Rolle von Karten für die literarische Imagination, die räumliche Ordnung und kulturelle Verortung fiktiver Welten und ihres Inventars oder auch die diskursive Funktion unterschiedlicher Raumkonzepte (etwa „glatte“ und „gekerbte“ Räume) für die Textstrukturierung untersucht haben (Foucault, 1990; Maresch und Werber, 2002; Weigel, 2002; Honold, 2004). Die Annahme, dass „Räume Kommunikationen strukturieren“, umgekehrt aber auch selbst „erst kommunikativ geschaffen“ werden (Geppert et al., 2005:18; vgl. Glasze und Pütz, 2007), wird von der im „spatial turn“ engagierten Literaturwissenschaft geteilt und spezifiziert: Räume strukturieren literarische Kommunikation, und Räume werden in literarischen Texten mit literarischen Verfahren geschaffen. Ein DFG-Symposion im Jahre 2004 hat die wichtigsten Ansätze der Literaturwissenschaften versammelt und die Relevanz der Raumdimension für die Analyse literarischer Texte vielfältig nachgewiesen (Böhme, 2005). Jörg Dünne und Robert Stockhammer haben mit ihren Monographien über die „Kartographische Imagination“ und die „Kartierung der Erde“ einen wichtigen romanistischen bzw. komparatistischen Beitrag zum Raum der Literatur vorgelegt (Dünne, 2011; Stockhammer, 2007). Der Verfasser dieses Beitrags hat an der Erschließung dieses For- 
schungsfeldes mitgewirkt (vgl. Joachimsthaler, 2005; Frank et al., 2008; Brill, 2011) vor allem hinsichtlich der Methodenkritik und mit Beiträgen zur Struktur und Semantik literarischer Raumentwürfe und ihrer Resonanz (Werber, 1998, 2007). Der vorliegende Beitrag untersucht vor diesem literaturwissenschaftlichen Hintergrund die literarische Verfasstheit geopolitischer Texte, um einen bislang nicht beachteten Aspekt der „Geopolitik avant la lettre“ in den Blick zu bekommen, der sich in der ,deutschsprachigen Geographie bis zum Ersten Weltkrieg“" (Schultz, 2001) nicht findet, sondern in der Geschichte der deutschsprachigen Literatur. Insbesondere narrative Texte und ihre Topographien, so möchte ich zeigen, gehören zur Vorgeschichte der deutschen „Rede über den Raum" (Köster, 2002) und geben ihr eine ganz bestimmte Ausprägung und Richtung.

Zweitens lassen sich zentrale Theoreme der Politischen Geographie in der Literatur des 19. Jahrhunderts freilegen, so dass man überpointiert sagen könnte, die „Geopolitik avant la lettre“ ist zuallererst tatsächlich Literatur. Bevor sich am Ende des 19. Jahrhunderts eine akademische Disziplin zu konstituieren beginnt, finden sich einige ihrer prominenten Lehrmeinungen und Grundüberzeugungen in literarischen Texten. Hier wäre eine Revision der Literaturgeschichte vonnöten, die die geopolitische Verfasstheit ihres Feldes bislang weitgehend übersehen hat. Beide Thesen zur „Geopolitik als Literatur" hängen eng zusammen, und ich möchte beide Thesen exemplarisch am Beispiel eines Romans von Gustav Freytag aus dem Jahre 1855 diskutieren, einem weit verbreiteten Roman, der literaturgeschichtlich als Muster des bürgerlichen Realismus gilt und der zu seinen Hunderttausenden von Lesern auch solche zählt, die die Geopolitik des Deutschen Reiches mitgestaltet oder exekutiert haben (vgl. Darré, 1940).

Zur Geschichte der Politischen Geographie gehört ein Präludium, das einerseits zu den romantischen Geographen wie Carl Ritter und zu evolutionistischen Soziologen wie Spencer führt, anderseits aber zu Autoren von Belletristik wie Gustav Freytag. In dessen überaus erfolgreichen (bereits am Ende des Jahrhunderts überschritt die Auflage die 100000 , 1925 sind es eine halbe Million Exemplare) Entwicklungs-, Kaufmanns- und Kolonialroman Soll und Haben (Freytag, 1953) sind geopolitische, anthropo-, verkehrs- und sozialgeographische Versatzstücke avant la lettre nachweisbar, mit denen der Text Räume, Bevölkerungen und Geschichten konstituiert, anhand markanter Eigentümlichkeiten unterscheidet und die so gewonnenen scharfen Distinktionen der „Raumschicksale" (Grabowsky, 1933) von Deutschen, Slawen und Juden aufgrund ihrer quasi ,natürlichen“, nämlich letztlich erdräumlichen und biologischen Ursachen legitimiert. Was man heute - selbst mit Sympathien für Ontologien (Schlottmann, 2013) - für hochvoraussetzungsvolle soziale Konstrukte halten würde, inszeniert Freytag als Effekte einer Natur, die so ist, wie sie ist. So wie Halford Mackinder 1904 das Nomadische als Funktion der eurasischen Steppe verstanden hat (Mackinder, 1998) oder so wie Alfred Kirchhoff 1905 die
Engländer, ihre Gesellschaft und ihr Empire aus der Insellage Britanniens erklärt (Kirchhoff, 1905), so werden in Soll und Haben bereits 1855 kulturelle und ethnische Unterschiede insbesondere zwischen Deutschen, Polen und Juden auf topographische Spezifika des Siedlungsraums, die daraus resultierenden unterschiedlichen Möglichkeiten seiner Aneignung und die davon abhängigen Verkehrs- und Kommunikationsbedingungen zurückgeführt. Umgekehrt wird im Roman die konkrete Ausgestaltung einer Landschaft auf ihre „Raumnahme“ (Schmitt, 1973) durch eine bestimmte Rasse zurückgeführt. Deterministisch ist dieser Diskurs nicht unbedingt, wohl aber evolutionistisch:

Allerdings wäre es geistlos pseudogeographischer Fanatismus, wollte man dieses Verhältnis wie einen naturgesetzlichen Zwang deuten. Der Mensch ist kein willenloser Automat; er verhält sich zu den Naturanregungen seiner Heimat bald wie ein gelehriger, bald wie ein teilnahmsloser Schüler. Das Wasser des heutigen Welthafens von Neuyork diente einst den Indianern bloß zum Sammeln essbarer Muscheln; an derselben Schärenküste, die die Norweger zu so kühnen Schiffern erzog, leben die Lappen weiter als armselige Fischer. (Kirchhoff, 1905:24)

Raum und Lage determinieren demnach keineswegs die Geschichte eines Volkes und die Politische Geographie ist mehr als „Geodeterminismus“ (Werlen, 2000:383), aber Raum und Lage stellen die "natürlichen Grundlagen“ der Evolution eines „Staates als Organismus“ (Ratzel, 1903:5; vgl. Kjellén, 1924:70f.), der sie entweder als Chance nutzt wie die USA und England oder sie in ihren Möglichkeiten verkennt, wie dies laut Kirchhoff die Indianer oder die Lappen getan haben. In der „Schule des Raums“, um eine evolutionistische Metapher Ratzels aufzugreifen, erweisen sich die einen als „,gelehrig“, die anderen bleiben ,zurück“ (Ratzel, 1903:371). „Schlechte Schüler“ sind dann irgendwann ,,verschwunden“, man sieht sie ,aussterben“ (Ratzel, 1903:389). Gerade diese Differenz nutzt bereits Freytags Soll und Haben für sein Narrativ einer deutschen Raumnahme slawischer Landstriche.

Denn es ist ein proto-geopolitischer wie protodarwinistischer Blick, der in diesem Roman die geographischen Begebenheiten zu Faktoren in einem selektiven Prozess verwandelt. Fünfzig Jahre bevor Rudolf Kjellén den Staat als politische Lebensform definiert und angesichts endlicher Räume und Ressourcen in einen evolutionären, bellizistischen Wettstreit mit anderen politischen Organismen schickt (Kjellén, 1916, 1917a, b, c, 1920, 1924), nimmt Soll und Haben nicht nur wegweisende Grundannahmen der Politischen Geographie Ratzels (,tellurische Auslese“, „Gesetz der wachsenden Räume“) vorweg, sondern gibt auch der künftigen deutschen Geopolitik, für die vor allem Karl Haushofer und sein Kreis stehen werden, eine Richtung 
vor, in die nach dem Ersten Weltkrieg das Deutsche Reich als Lebensform expandieren soll: gen Osten.

\section{Geopolitik ,avant la lettre“: Der Roman Soll und Haben}

Zunächst kurz zum Plot, soweit er für meine These relevant ist: Der Roman führt eine Gruppe von Personen von einem Landgut in Schlesien zu einem Besitz in Polen, das in eine Art deutsches Wehrrittergut verwandelt wird. Eine altadelige Familie, die Rothsattels, deren Oberhaupt anfangs seine Güter durch ein Majorat ewig an sein Geschlecht zu binden gedenkt, kapitalisiert die Güter, investiert das Geld und verliert den angestammten Besitz; ein junger, hanseatischer Edelmann, Fritz von Fink, erlernt einen bürgerlichen Beruf und wird Kaufmann, Überseehändler und Investor, spekuliert an der Wall Street und an der amerikanischen frontier mit Millionensummen, übernimmt ein überschuldetes Rittergut bei Krakau und baut es zu einem Vorposten deutscher Raumnahme aus. Zwei Knaben aus Ostrau, Veitel Itzig und Anton Wohlfahrt, steigen in den Handelshäusern Ehrenthal und Schröter auf, der eine immer diesseits, der andere auch jenseits von Recht und Moral. Für Wohlfahrt und Fink geht es gut aus, für Itzig und Rothsattel schlecht.

$\mathrm{Zu}$ den topographischen Details: Ort der Handlung ist zu Beginn des Romans ein äußerst gepflegtes, ertragreiches, ja schönes Landgut - es gehört den Freiherren von Rothsattel - an der Straße von Ostrau nach Breslau in der schlesischen Provinz des Königreichs Preußen zur Zeit der frühen 1830er Jahre. Die Gegend macht auf Freytags Protagonisten Anton Wohlfahrt, der von Ostrau in die Hauptstadt wandert, um eine Stelle beim Großhändler T.O. Schröter anzutreten, den allerbesten Eindruck.

Kleine Bäche von Erlen und Weidengruppen eingefaßt durchrannen lustig die Landschaft, jeder Bach bildete ein Wiesental, das auf beiden Seiten von üppigen Getreidefeldern begrenzt wurde. Von allen Seiten stiegen die hellen Glockentürme der Kirchen aus dem Boden auf, Mittelpunkt einer Gruppe von braunen und roten Dächern, die mit einem Kranz von Gehölz umgeben waren. Bei vielen Dörfern konnte man an der stattlichen Baumallee und dem Dach eines großen Gebäudes den Rittersitz erkennen, welcher neben den Dorfhäusern lag, wie der Schäferhund neben der wolligen Herde. (Freytag, 1953:10f.)

Diese Beschreibung ist äußerst aufschlussreich: Es ist eine gegliederte, durch Arbeit geschaffene Landschaft, in der ordnende Differenzen wie die von Zentrum und Peripherie, Feld und Flur, Schutz und Gehorsam unmittelbar augenfällig werden. Das Rittergut bewacht das Dorf wie der Schäferhund die Herde. Konzentrisch legen sich Dorf, Gehölz, Acker, Weideland um die Kirche. Bäche durchschneiden diesen Raum trennscharf, ohne Schnittmengen zu bilden wie Sümpfe oder Moraste, die Landstraßen sind vom Umland durch Gräben und Baumreihen markant unterschieden. Mit Carl Schmitt (Schmitt, 1997), der in Nomos der Erde aus dem Jahr 1950 eine Art Resümee der deutschen Geopolitik vorlegt, ${ }^{1}$ könnte man formulieren, der Zusammenhang zwischen „Ordnung und Ortung" werde in Freytags Roman anhand der Topographie dieser Landschaft unmittelbar augenfällig. Ihr genuiner Nomos offenbart sich nicht etwa nur in Gesetzen, Urkunden oder Verträgen, von denen Freytag ebenfalls ausführlich handelt (Werber, 2007:145ff.), sondern im Raum selbst, dessen Ordnung dieser Nomos geprägt hat bzw. dessen Ordnung er ist (Schmitt, 1973).

Nur das feste Land, nicht das Meer, taugt als Grund zivilisierter Ordnung, insofern es seine Bewirtschaftung durch den Menschen anzeigt und Einhegungen, Grenzsteine, Mauern trägt, jedenfalls „,feste Linien“ aufweist, die den Zusammenhang von „Ordnungen und Ortungen menschlichen Zusammenlebens offenkundig“" werden lassen (Schmitt, 1997:13). Die Landschaft, über die Anton Wohlfahrt kontempliert, ist nicht nur auf konkrete Weise situiert oder ,verortet“, nämlich an der Landstraße von Ostrau nach Breslau, sondern vermittelt dem Betrachter wie dem Leser durch ihre topographische Ordnung auch gleich die regionale Einrichtung des „,menschlichen Zusammenlebens“: Adel und Klerus herrschen patriarchalisch-pastoral über eine agrarische Bevölkerung, und die Natur segnet diese Ordnung mit ,üppigen“ Ernten. Selbstverständlich finden sich in der deutschen Literatur früherer Epochen ebenfalls extensive Landschaftsschilderungen, doch sind diese nicht geopolitisch konnotiert; eine systematische proto-geopolitische Aufladung literarischer Topographien finden sich bei Hölderlin (Albrecht, 1998) oder Kleist (Werber, 2008), im 17. Jahrhundert aber nicht.

Die Prägung der Erde durch Arbeit, durch Grenzziehung und durch Bauten - Deleuze und Guattari (Deleuze und Guattari, 1997) würden hier von Territorialisierung sprechen, der Erzeugung und Kontrolle eines „gekerbten“ Raums nennt Schmitt Landnahme (Schmitt, 1997:16f.). Die Gegend um das Gut des Barons Rothsattel führt in Soll und Haben das Ergebnis einer solchen Nahme vor; die aristokratischfeudale Ordnung hat sich der Topographie eingeschrieben. Mit der gleichen Evidenz, so wird zu sehen sein, werden Räume geschildert, die solch eine Ordnung bzw. überhaupt jedwede Ordnung vermissen lassen.

Im späteren deutschen Kolonialroman ist die geopolitische Annahme leicht nachzuweisen, der afrikanische oder

\footnotetext{
1 „Das Meer kennt keine solche sinnfällige Einheit von Raum und Recht, von Ordnung und Ortung. Zwar werden auch die Reichtümer des Meeres, Fische, Perlen und andere Dinge, von Menschen in harter Arbeit gewonnen“, schreibt Schmitt im Nomos der Erde, ,aber nicht, wie die Früchte des Erdbodens, nach einem inneren $\mathrm{Ma}$ von Saat und Ernte. In das Meer lassen sich keine Felder einsäen und keine festen Linien eingraben. Die Schiffe, die das Meer durchfahren, hinterlassen keine Spur. Auf den Wellen ist alles Welle.“(Schmitt, 1997:13)
} 
asiatische Raum sei von der einheimischen Bevölkerung nicht ,genommen“ oder „gekerbt“ worden (Frenssen, 1910; Benninghoff-Lühl, 1983; Honold, 2004) und insofern ungeordnet und unkultiviert, indes nur der ,gerodete und bearbeitete Boden“, der gekerbte Raum als Raum gelten könne, der die „Arbeit des Menschen“ und seine Ordnung erkennen lasse (Schmitt, 1997:13). Ein glatter Raum (Deleuze und Guattari, 1997:496, 499, 509ff.) dagegen wäre dementsprechend nicht nur ein recht- und ordnungsloser, sondern auch ein kulturloser, also unbearbeiteter und „ungepflegter“ Raum. Schmitts Begriff des Nomos enthält damit alles, was der Kolonialismus oder Imperialismus benötigt hat, um sich ein „,freies Feld“ der „Okkupation und Expansion“" zu imaginieren (Schmitt, 1997:55), dessen Bewohner notwendig unkultiviert und indolent sind, denn andernfalls hätten sie den Raum, den sie bevölkern, ja bearbeitet, geordnet, geprägt und gekerbt. Einen solchen „glatten Raum“ erfindet Gustav Freytag 1855 im Osten des deutschen Kulturraums. Der polnische Raum jenseits der preußischen Grenze wird von Anton Wohlfahrt denn auch ganz anders wahrgenommen als die schlesische Musterlandschaft:

An einem kalten Oktobertage fuhren zwei Männer bei dem Torgitter der Stadt Rosmin vorüber in die Ebene, welche sich einförmig und endlos vor ihnen ausbreitete. Anton saß in seinen Pelz gehüllt, den Hut tief auf der Stirn, neben ihm der junge Sturm im alten Reitermantel, die Soldatenmütze lustig auf einem Ohr. Vorn hockte auf einem Strohbund der Knecht eines Ackerbürgers und peitschte die kleinen Pferde. Der Wind fegte mit seinem riesigen Besen Sand und Strohhalme über die Stoppelfelder, die Straße war ein breiter Feldweg, ohne Gräben und Baumreihen, die Pferde wateten bald durch ausgefahrene Wasserpfützen, bald durch tiefen Sand. Gelber Sand glänzte zwischen dem dürftigen Grün der Äcker überall, wo eine Feldmaus den Eingang zu ihrer Grube angelegt, oder wo der emsige Maulwurf nach Kräften gearbeitet hatte, die Ebene durch kleine Hügelketten zu unterbrechen. In den Senkungen des Bodens stand schlammiges Wasser; an solchen Stellen streckten die ausgehöhlten Stämme alter Weiden ihre verkrüppelten Arme in die Luft, ihre Ruten peitschten einander im Wind, und die welken Blätter flatterten herunter in das trübe Wasser. (Freytag, 1855:493)

[...] Wieder wurden die Pferde eingespannt, wieder warfen sie ihre kurzen Beine im Sande vorwärts, und wieder ging es fort in der kahlen Gegend. Zuerst durch eine leere Ebene, durch einen schlechten Kiefernwald, dann über eine Reihe von niedrigen Sandhügeln, die wie Dünen der öden Wasserflut über den pflanzenarmen Boden hervorragten, dann auf schadhafter Brücke über einen kleinen Bach. „Hier ist das Gut“, sagte der Kutscher sich umdrehend, und wies mit der Peitsche auf einen Haufen dunkeler Strohdächer, welcher gerade vor ihnen sichtbar wurde. Anton erhob sich von seinem Sitz und suchte die Baumgruppe, in welcher das Herrenhaus liegen konnte. Er sah nichts davon. Um das Dorf war manches nicht zu finden, was auch die ärmlichsten Bauernhäuser seiner Heimat schmückte, kein Haufe von Obstbäumen hinter den Scheuern, kein umzäunter Garten, keine Linde auf dem Dorfplatz, einförmig und kahl standen die schmutzigen Hütten nebeneinander. (Freytag, 1855:497)

Der in den Literaturwissenschaften notorische „Realismus" Freytags ist alles andere als unschuldig (vgl. Wagner, 2006:18f.), entwirft sein Roman doch einen Raum, dem alles abgeht, was eine deutsche Kulturlandschaft auszeichnet, ganz als ob östlich der Oder die Kultur ende und die Wildnis beginne. Die schier endlose Ebene ist in der Raumkonstitution des Romans nahezu unstrukturiert, statt Straßen gibt es Feldwege, deren Bahnen sich im Sand oder im Schlamm verlieren. Der Kiefernwald erhebt sich über Äckern und Feldern wie „Dünen der öden Wasserflut“. Alles ist armselig, trüb, verkrüppelt, kahl oder leer. Aus der Grundannahme einer Einheit von Ordnung und Ortung könnte hier nur geschlossen werden, dass die Herrschaft genauso „schlecht“ sein müsse, wie es das Land selber ist, und genau diese Schlussfolgerung bietet der Roman an. Diese fatale Beschreibung des Ostens und ihre geopolitischen Implikationen sind zu einem Topos geworden, der noch in den im „Generalplan Ost“" manifestierten Raumvorstellungen nachzuweisen ist (Werber, 2012):

Eine Denkschrift für Hans Frank, dem Generalgouverneur des Generalgouvernements, beginnt einschlägig mit dem Kapitel „Polnische Wirtschaft“. Ihr Autor, Karl Kuchenbäcker, Leiter des sogenannten Hauptlandamtes der Regierung des Generalgouvernements, beschreibt dort den polnischen Raum aus der Perspektive eines Reisenden, der zum ersten Mal den Raum östlich der Reichsgrenze betritt:

Mancher, der zum ersten Male über die deutsche Grenze nach Osten fährt, kennt von Polen nicht viel mehr, als diese landläufige Redewendung. In mancherlei Gestalt bestätigen sich ihm nun seine Vorstellungen. Er flucht auf die schlechten Straßen und Wege, auf denen kein Fortkommen ist, ihn ärgern Tag und Nacht die Landstraße bevölkernden Kolonnen von Menschen und Fuhrwerken, die niemals abzureißen scheinen, er wundert sich über das magere Vieh, das in den Straßengräben, auf den Feldrainen oder an den Waldrändern herumbotanisiert, wo es doch nichts findet, und über Menschen, die dabeistehen, oft mehr als es Tiere sind, und die von morgens bis abends in den lieben langen Tag hineinträumen. Er sieht seitwärts des Weges auf die schmalen Ackerstreifen, die bald in die Fluren 
hinzufliehen scheinen, und bald in wirrem Durcheinander die Landschaft in tausende und kleinste Fetzen zerreißen. Sein Auge übt Kritik an der Ackerbestellung, an den Saaten und an der Ernte, an allem, am wenigsten vielleicht an dem braven Panje, der schlecht und recht hinter seinen vorsintflutlichen Ackergeräten einherstolpert. Er stellt fest, dass ein Dorf so unzulänglich ist wie das andere, und eine Holzkate so eng, dreckig und mit Menschen überfüllt, wie alle. Und diese Eindrücke findet er Tag für Tag wieder von neuem bestätigt. Er entsinnt sich, daheim nichts ähnliches gesehen zu haben. So prägt sich ihm das äußere Bild dieses Raumes und er fragt sich unwillkürlich, warum es so ist. Er sucht nach Erklärungen. (zit. n. Madajczyk, 1994:359)

Tatsächlich hat Polen 1938 mit seinen ,,vorsintflutlichen“ Mitteln und tagträumenden Landwirten 13453 Millionen Tonnen Getreide produziert und Lebensmittel exportieren können (Zgórniak, 2002:285). Wenn es auch sicher nicht derart industriell entwickelt war wie sein westlicher Nachbar, muss doch das von Kuchenbäcker gemalte „Raumbild“ als eine Fiktion angesehen werden, die nach Form und Inhalt geradewegs aus Freytags Roman stammen könnte. Dass der Raum anders sei - ,in tausende und kleinste Fetzen“ zerspellt -, statt wohlgeordnet; dass die Bevölkerung dieses Bodens weder sich, noch sein Vieh oder seinen Besitz in Ordnung halte; dass hier ,,polnische Wirtschaft" herrsche, sind Stereotype, die von der deutschen Literatur gepflegt worden sind. Der im Nationalsozialismus zur Kampfformel reüssierte Begriff der ,,polnischen Wirtschaft" wird von Gustav Freytag dreimal an entscheidender Stelle und mit einschlägiger Funktion verwendet (Freytag, 1855:674, 739; Freytag, 1872-1880:1073), was der Freytag-Forschung entgangen ist (Hahn, 2005:239). Die Formel steht für einen Raum, der von seiner Bevölkerung nicht kulturell angeeignet worden ist, weil sie sich auf diesem Raum nomadisch bewegt und ihm daher keine Ordnung einprägt. In der Topographie des Romans wird der polnische Raum entsprechend zum Meer oder zur Wüste, zu einem glatten und ungekerbten Raum (Werber, 2005). Nichts von dem, was Schmitt zum Nomos zählt, vermögen Wohlfahrt und Sturm - beides sprechende Namen: erst wird der Osten gestürmt, dann das Heil des Volkes befördert - bei ihrem Ritt in den Osten auszumachen (Schmitt, 1997:13). Auf dem ,glatten“ polnischen Boden, so zeigt der Roman, kann demnach gar kein Staat existieren, also auch keine Bürger und Behörden, sondern allenfalls „Banden“ (Freytag, 1855:277) und „Bandenführer“ (Freytag, 1855:278). Der Führer der polnischen Insurgenten wird vom Erzähler tatsächlich „Häuptling“ genannt (Freytag, 1855:288), und der Kaufmann Schröter führt diese zivilisatorische Insuffizienz umstandslos auf „,rassische“ Mängel zurück. Er behauptet: „Es gibt keine Rasse, welche so wenig das Zeug hat, vorwärtszukommen, und sich durch ihre
Kapitalien Menschlichkeit und Bildung zu erwerben, als die slawische.“ (Freytag, 1855:273)

Freytags Roman entwirft den Osten als ungeordneten, unkultivierten, wüsten Raum, der, ganz so wie vierzig Jahre später Ratzel es sehen wird, vom ,kolonisierenden Volk“ bislang ,nur inselförmig [...] durchsetzt“" ist (Ratzel, 1923:138). Als Anton Wohlfahrt das neue Gut der Rothsattels in Polen als Verwalter in Besitz nimmt, stellt der Erzähler fest: „Er war ausgesetzt, wie auf einer [... I Insel.“ (Freytag, 1855:499f.) Und über die Kolonisationsarbeit des Deutschordens im Mittelalter schreibt Freytag: ,[... ] im Osten lag das verkleinerte Ordensland wie eine Insel zwischen dem Meere und dem slawischen Gebiet." (Freytag, 1872-1880:724) Diese Inseln warten auf ihre Vernetzung durch deutsche „Pioniere" (Freytag, 1855:586, 654). Soll und Haben liest sich wie ein Kolonialroman, der deutsche Siedler in Steppen, Wüsten und Einöden schickt, um sie zu kultivieren, zu ordnen und anzueignen. Ratzel weist 1897 „Polen“ dann explizit als „Kolonialland“ aus (Ratzel, 1923:158, 138).

„Der Raum der Seßhaftigkeit wird durch Mauern, Einfriedungen und Wege zwischen den Einfriedungen eingekerbt, während der nomadische Raum glatt ist." (Deleuze und Guattari, 1997:524). Auch den Ozean zählen Deleuze und Guattari neben Wüste, Steppe und Eis zu den glatten Räumen (Deleuze und Guattari, 1997:526f.). Diese Unterscheidungen sind aber nicht nur für die geopolitische Semantik von großer Bedeutung, da sie ein ganzes Wissensfeld organisieren (vgl. etwa Schmitt, 1993), sondern auch für die Lektüre von Freytags Roman aufschlussreich, der im Osten Deutschlands einen glatten Raum ausweist, welcher als Meer, Wüste und Steppe inszeniert wird; Ordnungsräume in diesem glatten Raum entwirft der Text folgerichtig als „Inseln“ oder „Karawansereien“ (Freytag, 1953:294). Gustav Freytag hat vier Jahre nach dem Erscheinen seines Romans ein mehrbändiges populäres Geschichtswerk unter dem Titel Bilder aus der deutschen Vergangenheit (Freytag, 1927) publiziert, in dem er der „Besiedelung des Ostens“ ein eigenes Kapitel widmet. Der Deutsche habe die ,endlose polnische Ebene“ (Freytag, 1927:117) durch Stadtgründungen nach deutschem Recht (Freytag, 1927:116) wegbar gemacht. Von den ,,polnischen Städten“ behauptet Freytag, sie seien mit „deutschen Städten“ nicht zu vergleichen. Nichts sei hinter ihren Gräben zu finden, was hinter deutschen Stadtmauern selbstverständlich sei, von der Herrschaft des Rechts und der Sicherheit des Eigentums bis zur Tüchtigkeit, Sittsamkeit und Gelehrsamkeit der Bürger (Freytag, 1927:114). Daher sei alles, was man denn auf polnischem Boden an ,Wissenschaft, Verkehrsrecht und Kriegführung, Kunst und Handwerk“ antreffen möge, erst ,aus deutschem Land zu den Slawen gekommen“ (Freytag, 1927:109). Soll und Haben macht diese Unterscheidung von deutscher Raumordnung und slawischem Unvermögen mit allen Mitteln literarischer Suggestion evident. Der Raum im Osten wird so zu einem „,freien Feld“ der „Okkupation und Expansion“ (Schmitt, 1997:55), weniger erklärt als literarisch entworfen. Zugleich wird seine Ordnung und Kulti- 
vierung zu einer genuin deutschen Mission stilisiert. Freytag führt in Soll und Haben einen Narrationsstrang zur Kolonisierung Polens, dessen Rhetorik und Metaphorik für die Geopolitik der deutschen Weltkriege ebenfalls typisch sein wird. Der forcierte Rückgriff auf die Geschichte des Deutschordens in den 1920ern und 1930er Jahren findet sich ebenfalls bereits bei Freytag (vgl. Werber, 2012). Diese erste deutsche Raumnahme wird in Soll und Haben wiederholt und aktualisiert, wenn der Roman den Händler Wohlfahrt aus der Saturiertheit und Gemütlichkeit seines Kontors in Breslau in das revolutionäre Polen führt, ihn zu einer veritablen WohlFahrt auf einen Planwagen setzt (Freytag, 1855:493f.) und zum Ostlandfahrer (vgl. Venatier, 1939) umerzieht, der seinen im Kampf mit der feindlichen wie unwirtlichen Umgebung (,,polnische Wirtschaft“, ,slawische Wüste“) erfolgenden Gesinnungswandel so beschreibt:

Welches Geschäft auch mich, den einzelnen, hierhergeführt hat, ich stehe jetzt hier als einer von den Eroberern, welche für freie Arbeit und menschliche Kultur einer schwächeren Rasse die Herrschaft über diesen Boden abgenommen haben. (Freytag, 1855:624)

Die Arbeit, die in Polen verrichtet wird, ist für Freytag die gleiche, die der Deutsche Orden erst in Palästina, nach 1231 dann jenseits der Weichsel verrichtet habe: Der Raum wird gekerbt, territorialisiert, geordnet. Das Land wird vermessen und kultiviert (Freytag, 1953:535). Offenbar haben die slawischen Bewohner dies nicht vermocht. Das Landgut im Osten wird von Fink und Wohlfahrt belagerungsfest gemacht (Freytag, 1953:581) und erfolgreich gegen eine aufständische Bevölkerung in Überzahl verteidigt (Freytag, 1953:582f.).

Freytags Erzählung verwandelt einen biederen Geschäftsmann (Anton Wohlfahrt) und einen dandyhaften Adelssprössling (Fritz von Fink) in Eroberer und Kolonisten. Standes- und Klassenunterschiede werden unter den Kolonisten aufgehoben; vereint tritt eine deutsche - und nicht etwa preußische oder schlesische - Volksgemeinschaft in Polen einem zahlenmäßig überlegenen Feind auf Leben und Tod gegenüber. „Wir und die Slawen, es ist ein alter Kampf.“ (Freytag, 1855:624) Das Anwesen, das Fink und Wohlfahrt verteidigen, soll als Brückenkopf einer deutschen Raumnahme verstanden werden. Die Geschichte wiederholt sich:

Jetzt strömte die Volkskraft der Deutschen in vielen kleineren Wellen [...] nach Osten, und tausend Jahre nach der Auswanderung jener alten Germanen begannen die Thüringer und Sachsen an der Stromgrenze aufs neue den Kampf gegen die Fremden, mit stärkeren Waffen und festerer Kraft. (Freytag, 1953:719)

Anton Wohlfahrt und Fink errichten also in Polen einen „Wehrbauernhof“" nach dem Vorbild der alten „Rittergüter der mittelalterlichen ostdeutschen Kolonisation" (Madajczyk, 1994:8), gelegen inmitten der „slawischen Sahara“, wie Fritz von Fink formuliert, in der „Wüste“, wie Anton Wohlfahrt und Karl Sturm mehrfach feststellen (Freytag, 1855:629, 494, 441). Dass der Raum östlich von Schlesien und Pommern eine Wüste sei, die das Ergebnis der slawischen Verwüstung darstelle, ist eine Unterstellung, die sich später als prägend für die im Ersten Weltkrieg entstehende Konstruktion des Ostens erweisen wird (Werber, 2012). Zu den Topoi dieses Bildes des Ostens zählen die Vorstellungen, es handele sich um eine „Steppe“, eine „Wüste“ oder ein „Meer" (Liulevicius, 2002:189), jedenfalls aber um eine halt- und wurzellose „fürchterliche Leere“ (Liulevicius, 2002:190).

Polen sei „furchtbar verwüstet“, stellt Fritz von Fink in Soll und Haben fest, man könne aber ,etwas daraus machen" (Freytag, 1855:632f.). Dazu sind Fleiß und Arbeitswille nötig, die Freytag den Slawen durchweg abspricht. Wenn man erst einmal „etwas daraus“ gemacht hat, dann ist - im Roman! - der von der deutschen Kultur geprägte Raum durchaus als deutscher Raum zu erkennen (vgl. Jureit, 2012). Denn das Ergebnis der Raumnahme ist eine Kulturlandschaft, wie die Geopolitik sie nach dem Ersten Weltkrieg als Muster deutscher Raumordnung entwirft. Aber bereits Wohlfahrt und Fink verwandeln die slawischen Wüsteneien in „grüne Wiesen“ (Freytag, 1855:641). Überhaupt sei es ,,der Deutsche“, der in die polnische Gesetz- und Kulturlosigkeit „Ordnung einführt und einen bessern Zustand“ (Freytag, 1855:569). Für Freytag-Leser - und das sind Zehntausende - ist es dann geradezu eine Selbstverständlichkeit, wenn sie in den 1920ern lesen, der deutsche Kolonist betrete die slawische Wüste als „Kulturträger“ (Hampe, 1921:10). Es ist die Verwandlung der Wüste in eine Kulturlandschaft, die ihre Eroberung legitimiert, erklärt bereits Anton Wohlfahrt seinem Freund Fink mitten in Polen, weit entfernt von seinem Geburtsort Ostrau und seinem Wohnort Breslau:

„Und wer hat die große Landschaft erobert, in der ich geboren bin?", frug Anton weiter.

„Einer, der ein Mann war.“

„Ein trotziger Landwirt war's“, rief Anton, ,,er und andere seines Geschlechts. Mit dem Schwert oder durch List, durch Vertrag oder mit Überfall, auf jede Weise haben sie den Boden an sich gezogen, in einer Zeit, wo im übrigen Deutschland fast alles tot und erbärmlich war.“ (Freytag, 1855:625f.)

Was die „Ahnen“ einst taten, nämlich mit „Schwert“ oder „List“, durch „Vertrag“ oder „Überfall“ an „Boden“ zu gewinnen, um diesen Raum zu ordnen, das soll jetzt das ,junge“ und „,neue, deutsche Volk“ fortsetzen (Freytag, 1855:626). Fink versteht die Lehre, und der Erzähler kann am Ende von Soll und Haben das folgende Fazit ziehen:

Den Mann, welcher jetzt im Schloß gebietet, kümmert es wenig, ob eine Dohle schreit, oder die Lerche; und wenn ein Fluch auf seinem Boden liegt, er 
bläst $[\ldots]$ ihn hinweg. Sein Leben wird ein unaufhörlicher siegreicher Kampf sein gegen die finstern Geister der Landschaft; und aus dem Slawenschloß wird eine Schar kraftvoller Knaben herausspringen, und ein neues deutsches Geschlecht, dauerhaft an Leib und Seele, wird sich über das Land verbreiten, ein Geschlecht von Kolonisten und Eroberern. (Freytag, 1855:830f.)

\section{Geopolitische Fluchtlinien eines Romans}

Werke des sog. „bürgerlichen“ oder „poetischen“ Realismus (vgl. zu den Romanen dieser Epoche Plumpe, 1996) sind zur Genealogie der deutschen Politischen Geographie und Geopolitik zu zählen und, mehr noch, die Literatur schreibt an ihrer nach Beginn des Ersten Weltkriegs immer manifesteren politischen Programmatik mit. Freilich impliziert der bei Freytag geschilderte „Kampf“ um die (Rück-)Verwandlung der slawischen Wüste in eine deutsche Kulturlandschaft nicht das Konzept des Staates als politischer Organismus, dessen Evolution in der ökologischen Nische seiner geopolitischen Umwelt stattfindet. Es ist gegen Ende des Jahrhunderts der Zoologe Ratzel, der diese darwinistische und biologistische Komponente in die Politische Geographie einfügt, und erst Kjellén verhilft der bei Ratzel angelegten Staatsbiologie zu großer Resonanz (vgl. Werber, 2014). Zentrale Theoreme und Metaphern der Politischen Geographie finden sich aber, wie gesehen, bereits bei Freytag. Gewiss handelt Freytags Roman nicht von Staaten, sondern von Völkern. Aber wie bei Ratzel und Kjellén der Staat als Organismus stehen bei Freytag die Ethnien in einem zweifachen selektiven Verhältnis zur Umwelt: nämlich in Konkurrenz zu anderen Völkern und in Auseinandersetzung mit ihren ökologischen Nischen, an deren Gestalt, Ressourcen und Veränderungen sie sich anpassen und die sie zugleich verändern. Dieser Zusammenhang kennt zwei grundsätzliche Dimensionen - Raum und Zeit - und bedarf zu seiner Beschreibung der Geschichte wie der Geographie (Ratzel, 1903:25; Schlögel, 2003). Die Politische Geographie hat es dabei mit genau so großen Zeithorizonten zu tun wie Freytags epische Tiefe, die bis zu den Kreuzzügen zurückgeht. Diese Zeitdimension ist nötig, um die Entwicklung von Völkern und ihre Aneignung von Lebensräumen erzählen zu können. Vergleichbare Romane des 20. Jahrhunderts, etwa Hans Grimms Volk ohne Raum (Grimm, 1991), spannen ihre Narrative ebenfalls über Jahrhunderte.

Staaten seien „Organismen“ und „Großmächte biologische Tatsachen“; und ,aus eigener Lebenskraft und durch die Gunst der Konjunkturen, in ständigem Wettbewerb miteinander, also im Kampf ums Dasein und durch eine natürliche Auswahl stehen auch sie auf der Erdoberfläche da“, ist bei Kjellén zu lesen (Kjellén, 1924:37). Und bei Freytag: „Wir und die Slawen, es ist ein alter Kampf.“ (Freytag, 1855:624) Dieser Antagonismus findet in Soll und Ha- ben seinen geopolitischen Grund im Unterschied der Raumnahmen: Der Slawe bleibt bei Freytag Nomade in der Wüste, der Deutsche dagegen eignet sich den Raum an und verwandelt ihn in eine Kulturlandschaft. In der Politischen Geographie werden Darwins Schlüsselbegriffe immer wieder zitiert, universal competition, natural selection und struggle for existence (Darwin, 1859), und seine Evolutionstheorie geht in die Politische Geographie ein: Der „Staat“ sei „eine Art Leben“ und folglich auch den „Grundgesetzen des Lebens unterworfen“ (Kjellén, 1924:175). Aus der Sicht der Politischen Geographie wird „der Kampf ums Dasein im Grunde immer um Raum geführt“ (Ratzel, 1903:381). Die Antagonisten dieses Kampfes sind bei Ratzel wie bei Kjellén „Mächte“, „Völker“, „Nationen“ oder „Staaten“, die sich in Analogie zu Darwins Arten und Populationen den knappen „Lebensraum“ einer „Biosphäre“ (Ratzel, 1899:230) streitig machen. „Anthropogeographisch“ gesehen, ist dieser Krieg ein von „Staaten“ und „Völkern“ gegeneinander geführter „Kampf um Raum“ (Ratzel, 1899:244), dessen immer nur vorläufige Gewinner diese Räume für das eigene Wachstum besser (und zu Lasten der Konkurrenz) zu nutzen verstanden haben als die Verlierer. Genau dieses Verständnis findet sich bei Freytag. Die Entfaltung des einen hemmt oder versperrt die Entwicklung des anderen, aber alle streben nach Wachstum - nach Darwins Lebensgesetz ,all striving to increase“. Pflanzen, Tieren, Handelshäusern und Völkern ist dieses „Wachstum“ natürlich, und sie stoßen daher im Verlaufe der „Expansion“ früher oder später auf die hemmenden Kräfte konkurrierender Organismen oder Organisationen (Ratzel, 1903:130f.). Des einen Gewinn ist dann zugleich des anderen Verlust, der Wettbewerb der politischen Organismen wird als Nullsummenspiel aufgefasst. Auf dem ganzen „Erdkreis“ sieht der politische „Zoologe“ die einen „kleiner" werden, wenn die anderen ,wachsen“; dies sei ein von aller „Empirie“ bestätigtes „Gesetz“ (Kjellén, 1917b:5), das Gesetz ,allen Lebens“ (Ratzel, 1903:3). Anders hat es auch Freytag nicht gesehen. Deshalb können, wenn Fink, Wohlfahrt und die Deutschen gewinnen, Itzig, Rothsattel und die Polen nur verlieren. Der Grund dafür ist einerseits die „Lage“, also das „,beständige Verhältnis“ der Population ,,zur Erde" (Ratzel, 1903:259), anderseits aber die Population, die sich als ,guter“ oder ,schlechter“ Schüler des Raums erweisen kann. Die Slawen, so kann man Bilanz ziehen aus Soll und Haben, sind in der „Schule des Raums“ zurückgeblieben (Ratzel, 1903:371). Doch wird ihr „glatter Raum“ durch die Deutschen territorialisiert und gekerbt werden. Freytag weist bereits 1855 die polnischen Siedlungsgebiete als „Deutschen Osten“ aus und „begründet“ dies, anders als seine Zeitgenossen, die pauschal auf die kulturelle Unterlegenheit der Slawen hinweisen (Burleigh und Wippermann, 1991:25ff.), mit dem je spezifischen Verhältnis von „Rasse“ und „Raum“. Die Deutsche Geopolitik wird an diese fatale Semantisierung des Raums im Osten anschließen.

Der berüchtigte Generalplan Ost des NS-Regimes geht von der Annahme aus, die Völker und Nationen im „Os- 
ten“ könnten deswegen so leicht ,hinweggefegt" werden, weil sie sich als Nomaden den Raum nie angeeignet hätten. Der Reichsführer der Deutschen Bauernschaft des Dritten Reiches und Freytag-Leser Walther Darré hat 1933 die Slawen als parasitäre „Nomadenvölker“ bezeichnet und Soll und Haben für die Klarheit gelobt, mit der dort ,jüdisch-nomadisches und germanisch-bäuerliches Denken“ unterschieden werde (Darré, 1940:92). „Jüdisch-nomadisch“ meint in diesem Kontext wurzellos, ohne Verbindung von Volk und Boden - im Gegensatz zur deutschen Kulturlandschaft. Die Einwohner der ,,asiatischen Steppe“ seien ,,als Indianer zu betrachten“, erläutert Hitlers Reichsminister Todt in einem seiner Monologe am 17. Oktober 1941 (Madajczyk, 1994:24). Der Raum selber liefert den Rechtstitel zu seiner Eroberung, denn es handelt sich aus der Sicht Hitlers um einen Raum, den sich die zur Zeit dort befindliche Bevölkerung nicht angeeignet habe. „Hier im Osten werde sich zum zweiten Mal ein ähnlicher Vorgang wiederholen wie bei der Eroberung Amerikas“, führt Hitler in einem Tischgespräch des gleichen Tages aus (Madajczyk, 1994:23). Was es an Schönem im Osten gebe, sei ohnehin deutsch, der große Rest sei ,wüst und leer“ (Madajczyk, 1994:23), bevölkert von Nomaden und Indianern. „Es ist also nicht der Kolonist, der den Boden in Besitz nehmen will, sondern der Boden selbst, der nach dem Besessenwerden verlangt.“ (Kopp, 2005:231) Diese in der Semantik der Raumnahme immer wieder auftretende Denkfigur ist, wie Kristin Kopp gezeigt hat, typisch für den Kolonialroman, dessen Schauplatz Freytag in den Osten verlegt hat (Kopp, 2005:237).

Nach der ,militärisch-politischen Katastrophe des Weltkriegs“" (Preuss, 2008:221) kommt es zu einer Hochzeit geopolitischer Programme und Kampfbegriffe in Politik, Massenmedien, den Wissenschaften und der Literatur. Die geopolitische Semantik knüpft in vielerlei Hinsicht an die Denkformeln, Topoi und Metaphern der Politischen Geographie des Vorkriegs an, doch ist nach 1918 eine deutliche Neuausrichtung zu beobachten: Die Geopolitik nach der „Katastrophe“ gibt den vom kaiserlichen Imperialismus reklamierten „Platz an der Sonne“ (Bülow, 1907:7) und die Annexionsphantasien im Westen und Süden auf und orientiert sich nach Osten. Deutschland, so spitzt Peter Scherer zu, erlebt nach der Russischen Revolution von 1917

eine geopolitische Wende um 180 Grad. Drei Jahre zuvor hatte es einen Weltkrieg vom Zaun gebrochen, um seine maritimen Ansprüche gegen Frankreich und England geltend zu machen. Das Reich der Julikrise war ohne allen Zweifel nach Westen orientiert." (Scherer, 1989:47; vgl. zur alten „Hauptexpansionsrichtung“ ebd. 40; kursiv von NW)

Nach „Lebensraum“ wurde nun nicht mehr in den Kolonien oder entlang der Bagdadbahn (Dodds, 2007:33) gesucht, sondern im deutschen ,Hinterland“. Ludwig Dehio kommentiert dies 1952 so:
Welche Kehrtwendung vollzog sich damit! Der deutsche Imperialismus, der 1900 mit westlicher Blickrichtung ausgezogen war, um Englands Vormacht auf dem Ozean zu brechen - er gelangte jetzt zu einer östlich-kontinentalen Pointierung. (Dehio, 1952:499)

Der sogenannte „Deutsche Osten“ wird im Deutschen Reich zur neuen Leitformel des geopolitischen Denkens. Im Osten wird jener „Raum ohne Volk“ ausgemacht, den das „Volk ohne Raum“ benötige, um den angeschlagenen politischen Organismus zu heilen und auf einen Wachstumspfad zu setzen. Für diese Neuausrichtung der ,wachsenden Räume“ (Hesse, 1924) des Reiches in Richtung Osten sind mehrere Unterstellungen entscheidend, die bereits in Freytags Soll und Haben nachgewiesen worden sind und die allesamt auch in der Geopolitik oder bei geopolitisch beeinflussten Historikern der Zwischenkriegszeit virulent sind: (1) Der Osten ist schwächer besiedelt. (2) Der Osten ist von Slawen besiedelt. (3) Weil der Osten von Slawen besiedelt ist, ist er ein unkultivierter, wilder, ,glatter" Raum. (4) Im Falle einer Raumnahme durch ein zivilisatorisch überlegenes Volk würde der Osten aus einer Wüste oder Steppe in eine Kulturlandschaft deutscher Prägung und Ordnung verwandelt werden. (5) Die slawische Bevölkerung würde sich einer solchen Raumnahme nicht erfolgreich widersetzen können, weil sie kulturell unterlegen und nicht mit dem Boden verwurzelt ist. Diese fünf Grundannahmen der NS-Okkupations- und Geopolitik sind in Gustav Freytags Roman virulent.

\section{Schluss}

Die Lektüre des Romans bei „Geopolitikern“ der 1920er und 1930er Jahre ist bezeugt. Intertextuelle Analysen - wie im Falle Karl Kuchenbäckers - bringen eine so große Ähnlichkeit der Formulierungen und Denkmuster bei der Konstruktion des „Deutschen Ostens“ im Roman und in geopolitischen Texten zu Tage, dass es unwahrscheinlich erscheint, diese Übereinstimmungen dem Zufall zuzuschreiben. Jedenfalls scheint es mir lohnenswert zu sein, die Geschichte der Geopolitik über die Grenzen der ,deutschsprachigen Geographie“ hinaus zurückzuverfolgen und dabei literarische Quellen wie Freytags Roman Soll und Haben zu berücksichtigen. Gerade die geopolitische Umorientierung gen Osten nach dem Ersten Weltkrieg findet in der belletristischen Literatur ein klar konturiertes Vorbild. Dass ein literarischer Roman sich als anschlussfähig für geopolitische Texte erweist, wäre die eine, literaturgeschichtlich relevante Konsequenz, die aus den exemplarischen Analysen dieses Beitrages zu ziehen wäre. Die zweite Konsequenz ergäbe sich für die Wissensgeschichte der deutschen Geopolitik und ihrer Vorläufer in der Politischen Geographie: Die Oberflächenstruktur (die konkrete Ebene der Texte, die verwendeten Wörter, etwa „Steppe“ und „Wüste“) wie auch die Tiefenstruktur (ihre konstituierenden Differenzen und Denkbilder, 
ihre Argumentationsmuster und Deutungsusancen - etwa die Korrelation nomadischer Bevölkerungen und glatter Räume und die daraus abgeleitete Chance für Territorialisierungen) der Geopolitik weisen auf literarische Traditionen zurück. Um ihre konkrete Textgestalt, zumal im Haushofer-Kreis und unter den Autoren der Zeitschrift für Geopolitik, zu verstehen, lohnt sich eine Lektüre Gustav Freytags. Eine interdisziplinäre Erschließung des geopolitischen Diskurses innerhalb und außerhalb der geographischen Disziplin wäre sicher wünschenswert und ergiebig. Eine Grundlage dieser Zusammenarbeit könnte in der kulturwissenschaftlichen Ausrichtung gefunden werden, die die Geographie genauso bereichert hat wie die Literaturwissenschaften. Da die Semantik aktueller Themenfelder von der Migration bis zum Terrorismus, von den „Neuen Kriegen“ bis zum Klimawandel auch (!) literarisch wie räumlich konstituiert ist, wäre eine engere Zusammenarbeit zwischen Kulturgeographie und kulturwissenschaftlich orientierten Literaturwissenschaften wünschenswert. Es mag sein, dass das Ausrufen des „spatial turns" schon eine Weile her ist (vgl. Döring und Thielmann, 2008) und nicht alle radikalkonstruktivistischen Facetten dieser Wende überzeugen konnten, doch sind die interdisziplinären Forschungsmöglichkeiten, die der ,turn“ den Disziplinen eröffnet hat, noch lange nicht erkundet worden.

Datenverfügbarkeit. Für diesen Artikel wurden keine Datensätze genutzt.

Interessenkonflikt. Der Autor erklärt, dass kein Interessenkonflikt besteht.

Edited by: Benedikt Korf

Reviewed by: two anonymous referees

\section{Literatur}

Albrecht, C. V.: Geopolitik und Geschichtsphilosophie. 1748-1798, Akademie Verlag, Berlin, 1998.

Benninghoff-Lühl, S.: Deutsche Kolonialromane 1884-1914, Übersee Museum, Bremen, 1983.

Böhme, H. (Hrsg.): Topographien der Literatur, Metzler, Stuttgart, 2005.

Brill, H.: Vom Raum und seiner Bedeutung, Z. Polit., 58, 89-103, 2011.

Bülow, B. v.: Fürst Bülows Reden nebst urkundlichen Beiträgen zu seiner Politik, Herausgeber: Penzler, J., Reimer, Berlin, 1907.

Burleigh, M. und Wippermann, W.: The Racial State: Germany 1933-1945, Cambridge UP, Cambridge, 1991.

Darré, R. W.: Um Blut und Boden. Reden und Aufsätze, Zentralverlag der NSDAP, Franz Eher Nachf., München, 1940.

Darwin, C.: On the origin of species by means of natural selection, or the preservation of favoured races in the struggle for life, Murray, London, 1859.
Dehio, L.: Gedanken über die deutsche Sendung 1900-1918, Hist. Z., 174, 479-502, 1952.

Deleuze, G. und Guattari, F.: Tausend Plateaus, Merve, Berlin, 1997.

Dodds, K.: Geopolitics: A very short introduction, Oxford UP, New York, 2007.

Döring, J. und Thielmann, T. (Hrsg.): Spatial Turn. Das Raumparadigma in den Kultur- und Sozialwissenschaften), Transcript, Bielefeld, 2008.

Dünne, J.: Die kartographische Imagination. Erinnern, Erzählen und Fingieren in der Frühen Neuzeit, Fink, München, 2011.

Foucault, M.: Andere Räume, in: Aisthesis. Wahrnehmung heute oder Perspektiven einer anderen Ästhetik, Herausgeber: Barck, K., Gente, P., Paris, H., Richter, S., Reclam, Leipzig, 35-46, 1990.

Frank, M. C., Gockel, B., Hauschild, Th., Kimmich, D., und Mahlke, K.: Räume: Zur Einführung, Zeitschrift für Kulturwissenschaften, 2, 7-16, 2008.

Frenssen, G.: Peter Moors Fahrt nach Südwest. Ein Feldzugbericht, Grote'sche Verlagsbuchhandlung, Berlin, 1910.

Freytag, G.: Soll und Haben, Hirzel, Leipzig, 1855.

Freytag, G.: Die Ahnen, Hirzel, Leipzig, 1872-1880.

Freytag, G.: Debit and Credit, Harper, New York, 1858.

Freytag, G.: Bilder aus der deutschen Vergangenheit, 2 Bde., Leipzig, 1927.

Freytag, G.: Soll und Haben, Droemersche Verlagsanstalt, München, 1953

Geppert, A. C. T., Jensen, U., und Weinhold, J. (Hrsg.): Verräumlichung, in: Ortsgespräche. Raum und Kommunikation im 19. und 20. Jahrhundert, transcript, Bielefeld, 15-49, 2005.

Glasze, G. und Pütz, R.: Sprachorientierte Forschungsansätze in der Humangeographie nach dem ,linguistic turn“, Geogr. Z., 95, 14, 2007.

Grabowsky, A.: Raum als Schicksal. Das Problem der Geopolitik, Sonderdruck der „Zeitschrift für Politik“, Heymanns, Berlin, 1933.

Grimm, H.: Volk ohne Raum, Klosterhaus-Verlag, Lippoldsberg, 1991.

Hahn, H.: Die „Polenwirtschaft“ in Gustav Freytags Roman „Soll und Haben“. Studien zu Gustav Freytags kontroversem Roman, in: 150 Jahre „Soll und Haben“, Herausgeber: Krobb, F., Königshausen \& Neumann, Würzburg, 239-254, 2005.

Hampe, K.: Der Zug nach dem Osten. Die kolonisatorische Großtat des deutschen Volkes im Mittelalter, Teubner, Leipzig, Berlin, 1921.

Hesse, F.: Das Gesetz des wachsenden Räume, Zeitschrift für Geopolitik, 1, 1-4, 1924.

Honold, A.: Volk ohne Raum. Zur Imaginationsgeschichte der kolonialen Geographie, in: Kolonialismus, Herausgeber: Dabag, M., Gründer, H., und Ketelsen, U., Fink, München, 95-110, 2004.

Joachimsthaler, J.: Text und Raum, KulturPoetik, 5, 243-255, 2005.

Jureit, U.: Das Ordnen von Räumen. Territorium und Lebensraum im 19. und 20. Jahrhundert, Hambueger Edition, Hamburg, 2012.

Kirchhoff, A.: Mensch und Erde. Skizzen von den Wechselbeziehungen zwischen beiden, Teubner, Leipzig, 1905.

Kjellén, R.: Die Ideen von 1914. Eine weltgeschichtliche Perspektive, Hirzel, Leipzig, 1916.

Kjellén, R.: Die Großmächte der Gegenwart, Teubner, Leipzig, Berlin, 1917a. 
Kjellén, R.: Der Staat als Lebensform, Hirzel, Leipzig, 1917 b.

Kjellén, R.: Die politischen Probleme des Weltkriegs, 5 Ed., Teubner, Leipzig, Berlin, 1917c.

Kjellén, R.: Grundriss zu einem System der Politik, Hirzel, Leipzig, 1920.

Kjellén, R.: Der Staat als Lebensform, 4 Ed., Vowinckel, Berlin, 1924.

Kopp, K.: „Ich stehe hier als einer von den Eroberern“: „Soll und Haben“ als Kolonialroman, in: 150 Jahre „Soll und Haben“, Herausgeber: Krobb, F., Königskausen \& Neumann, Würzburg, 225-237, 2005.

Köster, W.: Die Rede über den „Raum“. Zur semantischen Karriere eines deutschen Konzepts, Synchron Verlag, Heidelberg, 2002.

Liulevicius, V. G.: Kriegsland im Osten. Eroberung, Kolonisierung und Militärherrschaft im Ersten Weltkrieg, Hamburger Edition, Hamburg, 2002.

Mackinder, H. J.: The Geographical Pivot of History, in: The Geopolitics Reader, Herausgeber: Tuathail, G., P. Routledge, London, 27-31, 1998.

Madajczyk, C. (Hrsg.): Vom Generalplan Ost zum Generalsiedlungsplan: Dokumente, Saur, München, New Providence, London und Paris, 1994.

Maresch, R. und Werber, N.: Permanenzen des Raums, in: Raum. Wissen. Macht, Herausgeber: Maresch Rudolf, N. W., Suhrkamp, Frankfurt am Main, 7-30, 2002b.

Plumpe, G.: Roman, in: Bürgerlicher Realismus und Gründerzeit. 1848-1890, Herausgeber: Plumpe, G., Hanser Verlag, München, 529-689, 1996.

Preuss, H.: Republik oder Monarchie? Deutschland oder Preußen, in: Gesammelte Schriften, Herausgeber: Lehnert, D. und Müller, C., Mohr Siebeck, Tübingen, 221-241, 2008.

Ratzel, F.: Anthropogeographie. Grundzüge der Anwendung der Erdkunde auf die Geschichte, Engelhorn, Stuttgart, 1899.

Ratzel, F.: Politische Geographie oder die Geographie der Staaten, des Verkehrs und des Krieges, 2 Ed., Oldenbourg, München und Berlin, 1903.

Ratzel, F.: Politische Geographie, 3 Ed., Oldenbourg, München und Berlin, 1923.

Scherer, P.: Freie Hand im Osten: Ursprünge und Perspektiven des Zweiten Weltkrieges, 3k-Verlag, Kösching, 1989.

Schlögel, K.: Im Raume lesen wir die Zeit. Über Zivilisationsgeschichte und Geopolitik, Hanser, München, 2003.

Schlottmann, A.: Reden vom Raum, der ist. Zur alltäglichen Notwendigkeit der Ontologisierung räumlicher Sachverhalte, in: Ontologien der Moderne, edited by: John, R., Rückert-John, J., und Esposito, E., Springer Fachmedien Wiesbaden, Wiesbaden, 189206, 2013.
Schmitt, C.: Nehmen/Teilen/Weiden. Ein Versuch, die Grundfragen jeder Sozial- und Wirtschaftsorsdnung vom Nomos her richtig zu stellen, in: Verfassungsrechtliche Aufsätze aus den Jahren 19241954. Materialien zu einer Verfassungslehre, Duncker \& Humblot, Berlin, 489-504, 1973.

Schmitt, C.: Land und Meer, Klett Cotta, Stuttgart, 1993.

Schmitt, C.: Der Nomos der Erde, Duncker \& Humblot, Berlin, 1997.

Schultz, H. D.: Geopolitik avant la lettre in der deutschsprachigen Geographie bis zum Ersten Weltkrieg, in: Geopolitik. Zur Ideologiekritik politischer Raumkonzepte, Kritische Geographie 14, Wien, 29-50, 2001.

Stockhammer, R.: Kartierung der Erde. Macht und Lust in Karten und Literatur, Fink Verlag München, 2007.

Venatier, H.: Vogt Bartold. Der grosse Zug nach dem Osten, Schwarzhäupter Verlag, Leipzig, 1939.

Wagner, B.: Verklärte Normalität. Gustav Freytags Soll und Haben und der Ursprung des „,Deutschen Sonderwegs“, Int. Arch. Sozialgesch., 20, 14-37, 2006.

Weigel, S.: Zum „topographical turn“. Kartographie, Topographie und Raumkonzepte in den Kulturwissenschaften, Kulturpoetik. Zeitschrift für kulturwissenschaftliche Literaturwissenschaft, 2, 151-165, 2002.

Werber, N.: Raum und Technik. Zur medientheoretischen Problematik in Luhmanns Theorie der Gesellschaft, Soziale Systeme, Zeitschrift für soziologische Theorie, 1, 219-232, 1998.

Werber, N.: Geopolitiken der Literatur. Raumnahmen und Mobilisierung in Gustav Freytags „Soll und Haben“, in: Topographien der Literatur. DFG-Symposion 2004, Herausgeber: Böhme, H., Metzler, Stuttgart, 456-478, 2005.

Werber, N.: Die Geopolitik der Literatur. Eine Vermessung der medialen Weltraumordnung, Hanser, München, 2007.

Werber, N.: Die geopolitische Erfindung von Volk und Reich. Heinrich von Kleists Hermannsschlacht als Gründungsmythos, in: Deutsche Gründungsmythen, Herausgeber: Galli, M. und PreuBer, H., Winter, Heidelberg, 91-104, 2008.

Werber, N.: Archive und Geschichten des „Deutschen Ostens“. Zur narrativen Organisation von Archiven durch die Literatur, in: Gewalt der Archive. Studien zur Kulturgeschichte der Wissenspeicherung, Herausgeber: Weitin, T. und Wolf, B., Konstanz University Press, Paderborn, 89-111, 2012.

Werber, N.: Geopolitik. Zur Einführung, Junius, Hamburg, 2014.

Werlen, B.: Sozialgeographie, 2 Ed., Haupt. UTB, Bern, Stuttgart, Wien, 2000.

Zgórniak, M.: Europa am Abgrund - 1938, LIT, Münster, 2002. 